\title{
EINE ÖKONOMISCHE ANALYSE DES MIGRATIONSRECHTS: WIE EFFEKTIV IST DIE FÖRDERUNG VON SOZIALRECHTEN FÜR ZUWANDERER IN BRASILIEN
}

\section{AN ECONOMIC ANALYSIS OF IMMIGRATION LAW: HOW EFFECTIVE IS THE PROMOTION OF SOCIAL RIGHTS FOR IMMIGRANTS IN BRAZIL}

\author{
Emília Lana de Freitas Castro \\ Universität Hamburg (Hamburgo, Alemanha) \\ Raphael Carvalho de Vasconcelos \\ Universidade do Estado do Rio de Janeiro - Uerj - (Rio de Janeiro, RJ, Brasil) \\ Universidade Federal Rural do Rio de Janeiro - UFRRJ - (Três Rios, RJ, Brasil)
}

Recebimento: 6 fev. 2017

Aceitação: 10 ago. 2017

\begin{abstract}
Como citar este artigo / How to cite this article (informe a data atual de acesso / inform the current date of access):
CASTRO, Emília Lana de Freitas; VASCONCELOS, Raphael Carvalho de. Eine ökonomische analyse des migrationsrechts: wie effektiv ist die förderung von sozialrechten für zuwanderer in Brasilien. Revista da Faculdade de Direito UFPR, Curitiba, PR, Brasil, v. 62, n. 2, p. 107 - 129, maio/ago. 2017. ISSN 2236-7284. Disponível em: $<$ http://revistas.ufpr.br/direito/article/view/50515>. Acesso em: 28 ago. $2017 . \quad$ DOI: http://dx.doi.org/10.5380/rfdufpr.v62i2.50515.
\end{abstract}

\section{KURZZUSAMMENFASSUNG}

In diesem Artikel geht es um die Analyse der Migrationskosten in Brasilien, insbesondere um die Kosten, die dem brasilianischen Staat entstehen, wenn er Sozialrechte für Zuwanderer fördert. Nachdem die Arbeit mit den wichtigsten Definitionen und mit den Erklärungen der Grundbegriffe eröffnet wird, wird der Zugang von Migranten zu den Sozialrechten in Brasilien erläutert. Ziel dieser Arbeit ist aufzuklären, ob und wie eine ökonomische Analyse des Migrationsrechts - insbesondere des Sozialrechts - in Brasilien möglich ist und was die Folge von solch einer Recherche für die Planung einer Migrationspolitik wäre. Die relevantesten Rechtsvorschriften, die die Gesundheit, die Sozialhilfe und die Ausbildung betreffen, werden dargestellt und der Anspruch von Zuwanderern auf diese Rechte werden gezeigt. Diese Arbeit konzentriert sich insoweit auf die folgenden Fragen: (1) ob es ein passendes Modell für eine ökonomische Analyse des Migrationsrechts im Rahmen des Sozialrechts für Migranten gibt, (2) wenn dies der Fall sein sollte, wie dieses Modell aussehen könnte, und (3) inwiefern dieses vorgeschlagene Modell in Brasilien anwendbar ist.

\section{STICHWÖRTER}

Migrationsrecht. Ökonomische Analyse des Rechts. Brasilien. Sozialrechte.

\section{ABSTRACT}

This article deals with the analysis of the cost of migration in Brazil, in particular the costs incurred by the Brazilian state when it promotes social rights for immigrants. This research is developed under a context of intense human mobility in the world, during which Brazil plays an important role, as still one of the most attractive countries for immigrants nowadays. The aim of this work is to clarify 
whether and how an economic analysis of immigration law - especially social law - is possible in Brazil and which would be the result of such a research for the sake of a well planned migration policy. After the most important points on the right to health, to welfare and to education are presented, it follows a detailed explanation about the access of immigrants to these rights. This work focuses on the following questions: (1) whether there is a suitable model for an economic analysis of migrant law in the context of social law for migrants, (2) if this is the case, how should this model look like, and (3) to what extent this proposed model is applicable in Brazil.

\section{KEYWORDS}

Migration law. Economic analysis of law. Brazil. Social rights.

\section{EINLEITUNG}

Das Thema Migration sorgt heutzutage für heftige Diskussionen und Überlegungen. Die Naturkatastrophen, die Suche nach besseren Arbeits- und Lebensbedingungen und die Globalisierung im Allgemeinen sind nur einige der Gründe warum es zu immer stärker werdenden Migrationsbewegungen kommt. Nach Schätzung der Vereinten Nationen (2013, 2) leben heute rund 3,2\% der Weltbevölkerung in Ländern, die nicht ihre ursprüngliche Heimat sind. Noch nie waren Menschen so oft unterwegs und so mobil wie in der jetzigen Zeit.

Die Aufnahme von Migranten spielt daher eine immer wichtigere Rolle in der Politik, in den Sozialwissenschaften und auch im Recht. Der Zuwachs von Einwanderern auch in ärmeren Ländern der Welt ist eine Tatsache und stellt die klassische Bewegung der Menschen, die von ärmeren Ländern in reichere Länder auswandern zunehmend in Frage. In dieser Arbeit wird die Rolle des Schwellenlandes Brasilien hervorgehoben, denn das größte Land Südamerikas zieht immer mehr Migranten an.

Im Hinblick auf eine ökonomische Perspektive des Migrationsrechts in Brasilien, sollen in dieser Arbeit einige Fragen beantwortet werden. Diese Arbeit konzentriert sich insoweit auf die folgenden Fragen: (1) ob es ein passendes Modell für eine ökonomische Analyse des Migrationsrechts im Rahmen des Sozialrechts für Migranten gibt, (2) wenn dies der Fall sein sollte, wie dieses Modell aussehen könnte, und (3) inwiefern dieses vorgeschlagene Modell in Brasilien anwendbar ist. Das bedeutet, dass diese Arbeit sich auf die Migrationskosten konzentriert. Die Darstellung der Recherche basiert sowohl auf der Analyse von Daten, die von der brasilianischen Regierung zur Verfügung gestellt wurden, als auch auf Literatur aus den ökonomischen und rechtlichen Studien, die grundsätzlich das Thema der Migration umfassen. Wichtig ist es zu erwähnen, dass diese Arbeit von der ökonomischen Analyse des Migrationsrechts wesentlich von der Betrachtung des Sozialrechts geprägt ist, denn (a) die angebotenen Sozialleistungen sind ein gewichtiger Grund für Einwanderer, 
wenn sie sich für das Leben in einem spezifischen Land entscheiden und weil (b) nur durch aktives staatliches Handeln die Verwirklichung solcher Sozialleistungen ermöglicht wird.

Die vorliegende Arbeit beginnt mit den wichtigsten Definitionen und mit den Erklärungen der Grundbegriffe. Danach wird der Zugang von Migranten zu den Sozialrechten in Brasilien erläutert. Die bisher aufgezeigten Punkte zielen nicht nur auf die Analyse der Nutzen und Kosten des brasilianischen Staates bei der Versorgung von Sozialrechten für Zuwanderer ab, sondern auch auf den Vorschlag eines Modells, das eine objektive Analyse des Migrationsrechts bzw. des Sozialrechts für Migranten ermöglicht. Abschließend lässt sich feststellen, ob und wie eine ökomische Analyse des Migrationsrechts in Brasilien möglich ist und was die Folge von so einer Recherche für die Planung einer Migrationspolitik wären.

\section{DEFINITIONEN UND ERKLÄRUNGEN DER GRUNDBEGRIFFE}

Aus Gründen der besseren Verständlichkeit der Arbeit werden in einem ersten Schritt die wesentlichen Hauptausdrücken im Rahmen der ökonomischen Analyse des Migrationsrechts erklärt und definiert.

\section{1 ÖKONOMISCHE ANALYSE DES MIGRATIONSRECHTS}

Der Ausgangsüberlegung für eine ökonomische Analyse des Migrationsrechts knüpft an das Wachstum der reicheren Länder und an der Stagnation in den ärmeren Ländern an. In anderen Worten: Migranten wandern von ärmeren Ländern in reichere Länder aus - die sogenannte „one-wayissue” (Eger u. Weber 2014, 6) - und diese internationale Bewegung ist eine ökonomische Reaktion auf die Einkommenskluft: je tiefer diese Kluft zwischen den Ländern ist, desto größer wird der Druck auszuwandern (Collier 2015, 44). Außerdem stellt Paul Collier in diesem Zusammenhang fest, dass die Migration zu einer Investition wird, denn es gibt eine Vielzahl von „- ökonomische[n], rechtliche[n] und soziale[n] -,, Migrationshindernissen (Collier 2015, 44-45). Das heißt, die Auswanderungswünsche werden erfüllt, indem diese (auch ökonomische) Hindernisse einigermaßen erfolgreich überwunden werden.

Darüber hinaus hängt die Migrationsrate auch von den Einwanderergemeinden in den Aufnahmeländern ab, d. h. die bestehende Anzahl von Migranten in den Aufnahmeländern ist von großer Bedeutung, inwieweit sich die Migranten für die Auswanderung in dieses bestimmten Land entscheiden (Collier 2015, 44-45). 
Weil die Migration von vielen ökonomischen, sozialen und rechtlichen Variablen abhängig ist, erscheint es sinnvoll sich der ökonomischen Analyse des Migrationsrechts zu widmen.

Dabei ist anzumerken, dass Migration allgemein als einer der wichtigsten Strukturfaktoren der globalen Wirtschaft anerkannt ist. Die wechselseitigen Zusammenhänge zwischen (1) ökonomischen Zyklen, (2) demografischem Wandel und (3) Migration haben entscheidenden Einfluss auf den (globalen) Arbeitsmarkt: Sie sind verantwortlich für den Bedarf an und für den Überschuss von Arbeitskräften und auch für die steigende bzw. abnehmende Nachfrage nach verschiedenen beruflichen Fähigkeiten und Qualifizierungen. Sie sind darüber hinaus auch wichtige Faktoren für das Verständnis des Verhältnisses zwischen der globalen Verteilung von erwerbstätigen und nicht erwerbstätigen Menschen (International Organization for Migration 2012, 19): Für die Befriedigung der Nachfrage nach bzw. dem Abbau von Überschüssen an Arbeitskräften ist Migration ein Ausgleich bzw. ein regulierender Mechanismus und kann darüber hinaus - durch den Austausch (den Zu- bzw. Abfluss) von Talenten und Fachwissen - sogar für die gesamtökonomische Lage eines Landes entscheidend sein (International Organization for Migration 2012, 20).

Ziel einer ökonomischen Analyse des Migrationsrechts wäre es, die bestimmten ökonomischen Perspektiven von rechtlichen Hindernissen und/oder rechtlichen Maßnahmen der Migration in Betracht zu ziehen. Inwieweit die Einführung spezifischer rechtlichen Maßnahmen das Leben der Migranten beeinflussen kann und wie die Kosten und Nutzen sowohl des Staates als auch der Migranten durch eine Migration aussehen, soll durch die ökonomische Analyse des Migrationsrechts beantwortet werden.

Der ökonomischen Literatur zufolge ${ }^{1}$ kann es festgestellt werden, dass die Personenfreizügigkeit, die Niederlassungsfreiheit und Arbeitnehmerfreizügigkeit eine entscheidende Rolle für die ökonomische Analyse des Migrationsrechts spielen: Ein Land könnte von einer liberalen oder restriktiveren Migrations- und Niederlassungspolitik ökonomisch profitieren oder die Kluft zwischen wohlhabenderen und ärmeren Menschen vergrößern (Încalţărău et al. 2014, 285). Auch Flüchtlinge in Empfang zu nehmen und politische und rechtliche Maßnahmen für oder gegen das Beherbergen dieser Menschen zu entwickeln gehört nicht nur zum politischen Leben eines Landes, sondern wirkt sich auch auf seine Wirtschaft aus.

Ein anderer, ebenfalls sehr aufschlussreicher und bislang noch nicht erfasster Blickwinkel auf die ökonomische Analyse des Migrationsrechts könnte durch die von Cox und Posner (2007, 809-

\footnotetext{
${ }^{1}$ Wie, zum Beispiel: Eger, Thomas; Weber, Franziska: Immigration law, in: Encyclopedia of Law and Economics, S. 1; Sykes, Alan O.: The Welfare Economics of Immigration Law, S. 1-46; Brücker, Herbert; Eger, Thomas: The law and economics of the free movement of persons in the European Union, S. 146 - 179.
} 
856) vertretenen Theorie zu den „first-order policy preferences” und „second-order institutional design” eröffnet werden. Laut der Vorläufer der law and economics-Bewegung, bezeichnen die „firstorder policy preferences” die Ziele eines Staates, wenn er den Eintritt von bestimmten Migranten ins Territorium verhindern möchte. Diese Ziele umfassen einige Aspekte, wie zum Beispiel: Wie viele Migranten dem Land beitreten dürfen (quantity); welche Art von Menschen zuwandern dürfen (type); und unter welchen Bedingungen die Zuwanderer zugelassen werden dürfen (terms of admission) (Cox u. Posner 2007, 814-819).

Infolgedessen kann es unter „second-order institutional design” verstanden werden, wie das Sortieren von Migranten gemacht wird, sodass die sogenannten „first-order policy preferences” erreicht werden können (Cox u. Posner 2007, 824). Durch das „second-order institutional design” kann sich das Aufnahmeland folglich entscheiden, wie viele Zuwanderer welcher Qualifikation es unter welchen Umständen aufnimmt. Dadurch hat es Einfluss auf das Wachstum und auf die generelle Entwicklung der eigenen Ökonomie und kann die Entwicklung seines Arbeitsmarktes steuern (z. B. durch die Aufnahme hochqualifizierter Arbeitskräfte um bestimmte Bedürfnisse der Industrie zu befriedigen).

Weil die ökonomische Analyse des Migrationsrechts ein umfassendes Thema ist, konzentriert sich diese Arbeit auf die Kosten und Nutzen eines Staates (in diesen Fall des brasilianischen Staates) bei der Zuerkennung bestimmter sozialer Rechte an Migranten.

\subsection{MIGRATION UND MIGRANTEN}

Der Begriff Migration bezeichnet die Bewegungen von Menschen und Bevölkerungen zwischen Staaten und Kontinenten (Clarke 2011, 1). Die Vereinten Nationen haben die Definition von Migration so dargestellt, dass sie gleichzeitig die Definition von „internationalen Migranten” enthält: der Vereinten Nationen zufolge sind internationale Migranten alle Menschen, die ihren gewöhnlichen Wohnsitz von einem Land zu einem anderen ändern, und die aus diesem Grund Wanderungsbewegungen ausführen (United Nations Statistics Division 1998, § 32).

Gemäß Kälin und Künzli (2008, 577) können diese Bevölkerungsbewegungen freiwillig oder gezwungenermaßen und innerhalb des eigenen Landes oder über Landesgrenzen hinweg erfolgen. Aus dieser Beschreibung lassen sich die Charakteristika für vier Gruppen von Menschen ableiten, die menschen- und migrationsrechtlichen Schutzes bedürfen:

(1) Menschen, die sich frei entscheiden, ihren Wohnort aus ökonomischen, familiären oder anderen Gründen zu verlassen, und sich an einem anderen Ort im eigenen Land niederlassen; 
(2) Migrantinnen und Migranten, die aus den gleichen Gründen in ein anderes Land ziehen; (3) Binnenvertriebene, d. h. Menschen, die zum Verlassen ihres Wohnortes gezwungen werden und in einem andern Teil des eigenen Landes Zuflucht suchen [...]; und (4) Flüchtlinge, die gezwungen sind, im Ausland Schutz vor Verfolgung oder Vertreibung zu finden. (Kälin und Künzli 2008, 577)

Thomas Straubhaar $(1988,47)$ widmet sich in seinem Werk On the Economics of International Labor Migration insbesondere der internationalen Migration von Arbeitskräften und definiert diese als eine räumliche, soziale und nicht erzwungene Bewegung von Menschen von einem Land in ein anderes (sowie auch von einem Sozialsystem in ein anderes). Laut Straubhaar verlassen diese Migranten ihre Länder aus ökonomischen Gründen und beabsichtigen länger als ein Jahr im Ausland zu bleiben.

Obwohl die oben gegebenen Definitionen verschiedene Blickwinkel darstellen, ist es nicht schwierig festzustellen, dass die internationale Migration eine Bewegung von Menschen bezeichnet. Diese Bewegung von Menschen, die aus einem Land in ein anderes gehen, kann freiwillig oder unter Zwang erfolgen. Zwar kann es aus den gleichen Gründen auch zu Migration innerhalb eines Landes kommen, jedoch widmet sich diese Arbeit speziell den Problemen der internationalen Migration. Deswegen soll der Begriff „Migration” im Folgenden im Sinne von „internationaler Migration” begriffen und verwendet werden ${ }^{2}$.

Die Migration ist ein komplexes Phänomen, das sowohl politische als auch juristische und wirtschaftliche Aspekte hat. Sei es als ein ökonomisches und soziales Problem oder als ein wirtschaftlicher Vorteil können beide Gastland und Herkunftsland das Phänomen der Migration unter mehreren Perspektiven betrachten (Leão 2013, 97). Aus diesem Grund haben verschiedene Länder verschiedene Einstellungen gegenüber Migration und entwerfen unterschiedlich geprägte Migrationsrechtsvorschriften. Ziel- und Herkunftsländer erlassen daher Gesetze und Regelungen, die oft nicht nur dazu gedacht sind, Migranten zu beschützen, sondern auch ihr Leben im Gastland unter einigen Gesichtspunkten einzugrenzen und zu reglementieren. Diese Begrenzungen sind meistens erheblich einschneidender und strenger als jene des internationalen Investitionsrecht oder des internationalen Handelsrechts - wie z. B. solche, die den Verkauf von bestimmten Waren in einer spezifischen Region oder in einem entsprechenden Land betreffen.

Schließlich, um die Begriffe Migration und Migranten in Betracht zu ziehen, wird der Unterschied zwischen Migranten und Ausländer kurz erklärt. Ausländer zu sein bedeutet, nicht eine bestimmte Staatsangehörigkeit zu besitzen (Lopes 2009, 31). Laut Carmen Tiburcio (2001, 1) bedeutet das, dass alle, die eine bestimmte Staatsangehörigkeit nicht besitzen, innerhalb des

\footnotetext{
${ }^{2}$ Oft in dieser Arbeit aus praktischen Gründen nur als “Migration” bezeichnet.
} 
entsprechenden Landes als Ausländer anzusehen sind. Auf der anderen Seite sind die Migranten diejenigen, die Migrationsbewegungen ausführen, seien es Ein- oder Auswanderungen. Einwanderer sind demnach Ausländer, aber nicht alle Ausländer sind automatisch Migranten bzw. Einwanderer. Touristen zum Beispiel fallen unter die Kategorie “Ausländer”, sind aber keine Einwanderer, da sie sich nicht dauerhaft in einem fremden Land aufhalten. Einwanderer zu sein ist eine soziale Situation, während Ausländer zu sein einen rechtlichen Zustand bezeichnet (Lopes 2009, 32).

\subsection{MIGRATIONSKOSTEN}

Die Migrationskosten setzen sich aus verschiedenen sozialen, kulturellen und ökonomischen Faktoren zusammen und bilden zusammengenommen die Grundlage für oder gegen die Entscheidung, ein- oder auszuwandern. Weil diese Entscheidungen von großer ökonomischer Bedeutung sind (Bayer u Juessen 2012, 377), werden Nutzen und Kosten der Migration im Folgenden kurz erläutert. Dabei ist zu beachten, dass nicht nur die Entscheidung für die Migration auf Grundlage der Migrationskosten getroffen wird, sondern dass sich durch die Entscheidung, in ein anderes Land ein- bzw. aus einem Land auszuwandern, auch Rückschlüsse auf die die ökonomischen Situation der Sende- und Aufnahmeländer ziehen lassen. (Thomas Straubhaar 1988, 131)

Grundsätzlich lässt sich sagen, dass die Analyse des Nutzens und der Kosten einer Migration aus drei Prozessschritten besteht: Der erste Schritt wäre, die wichtigsten Nutzen und Kosten zu identifizieren; im zweiten Schritt kommt es dann zu einer Quantifizierung (monetären Betrachtung) der Kosten und Nutzen. Im dritten und letzten Schritt soll dann, laut Thomas Straubhaar, folgendes geschehen: "[...] the economic components of present and future streams of costs and benefits have to be discounted to their present value, and summed up. [...]”. (Thomas Straubhaar 1988, 131)

Ziel der vorliegenden Arbeit ist es allerdings, nur die ersten beiden Schritte dieser dreigliedrigen Betrachtungsweise auf das Fallbeispiel Brasilien anzuwenden, um die Kosten und die Nutzen von Migration auf das brasilianische Sozialsystem abmessen zu können - wobei zu beachten sein wird, dass die Kosten [sowohl auch Nutzen] auch in nicht-monetärer Form auftreten können (Michaelides 2011, 133).

\subsubsection{Nutzen und Kosten}

Wie oben bereits erwähnt wurde, ist es möglich, dass die Kosten und Nutzen der Migrationsbewegungen als monetärer oder als nicht-monetärer Vermögenswert betrachtet werden 
können. Die Nutzen und Kosten werden in diesem gemeinsamen Punkt dargestellt, weil viele Faktoren der Migration gleichzeitig als Nutzen und auch als Kosten betrachten werden können ${ }^{3}$.

Laut Michaelides $(2011,133)$ sollen die Kosten, denen ein Wert zugeschrieben werden kann, nur mit der Wanderung bzw. mit der Migration assoziiert werden, d. h. dass diese Kosten normalerweise nicht nach dem Beginn des geregelten Lebens im Ausland fortbestehen. Auf der anderen Seite sind die nicht-monetären Kosten jeder Zeit zu beobachten, auch nachdem die Migranten sich schon im Aufnahmeland eingelebt haben. Ein gutes Beispiel für solche andauernde Kosten sind die sozialen Kontakte, die im Aufnahmeland oder in einer bestimmten Region zu finden sind. Migranten, die in ihren Regionen verbreitete soziale Kontakte haben, würden nur mit geringer Wahrscheinlichkeit aus dieser Region fortziehen (Michaelides 2011, 133).

Auch ganz andere Faktoren wie der Vergleich zwischen den Wohnungskosten und dem Durchschnittslohn im Aufnahmeland spielt eine Rolle bei den Migrationsbewegungen und zählen zu den Migrationskosten (Bayer u Juessen 2012, 377). Die Komplexität der Migrationskosten ist daher nicht von der Hand zu weisen: Migrationskosten bestehen nicht nur aus hohen (Kosten) oder niedrigen (Nutzen) Wohnungskosten, oder aus starken (Nutzen) oder schwachen (Kosten) sozialen Kontakten. Zu den Migrationskosten zählen auch Gebühren für die Verwaltungstätigkeiten, die Pflichten der Migranten gegenüber dem Aufnahmeland (insbesondere die Steuerschuld) (Sykes 1992, 18), die aktuelle politische Lage im Aufnahmeland, die Tatsache, dass die Kontakte im Herkunftsland verloren gehen, und die relativ lange Zeit und die Anstrengungen, die erbracht werden müssen, um eine neue Sprache zu lernen. Die Anpassung an eine neue Kultur und an eine neue Umgebung, genauso wie die beste Entscheidung zu treffen, zu welcher Schule die Kinder gehen sollen, können auch bei den Migrationskosten nicht außer Acht gelassen werden (Eger u Weber 2014, 2).

Wenn alle diese Kosten und möglichen Schwierigkeiten in Kauf genommen werden, können aus einer Auswanderung aber auch positive Effekte (Nutzen) für die Auswanderer erwachsen; beispielsweise in Form von Sozialleistungen wie dem Kindergeld, Mietkostenzuschüssen, Arbeitslosengeld oder sogar der kostenlose Zugang zu Schulen und Universitäten (Eger u Weber 2014, 2). Auch andere Zustände sollen in diesem Sinne betont werden, wie z. B. die hohe oder niedrige Kriminalitätsrate, die Gewalttätigkeit in dem Aufnahmeland oder einfach die Rolle, die die Religion in der Gesellschaft des Aufnahmelandes spielt.

\footnotetext{
${ }^{3}$ Beispielsweise: Verbreitete soziale Kontakte sind für die Anpassung der Migranten von großer Bedeutung (Nutzen), während sehr geringe soziale Kontakte und wenige Kontaktverknüpfungen ein Grund für das Zurückkehren der Migranten sein können.
} 
Nichtsdestotrotz gibt es auch Fälle, in denen die Migranten keine Gelegenheit haben, über die Kosten oder Nutzen nachzudenken: Flüchtlinge, die um ihr Leben kämpfen und flüchten müssen und daher nach einem Land suchen, in dem sie ein menschenwürdiges Leben führen können, haben für gewöhnlich keine Gelegenheit die Entscheidung für ihre Auswanderung bzw. Flucht unter Einbeziehung dieser Gesichtspunkten zu fällen. Auch handelt es sich hierbei um einen Sonderfall, da die Staaten hier die Kosten der Migration übernehmen.

Bemerkenswert sind in diesem Sinne die Kosten und Nutzen der Länder: Aufnahmeländer gewinnen an Arbeitskraft durch die Migranten während Sendeländer z. B. von den sogenannten remittance flows ${ }^{4}$ profitieren, d. h., von den Überweisungen von Migranten an Familienmitglieder in der Heimat (Încalţărău et al. 2014, 286). Obwohl einige Migranten nicht zu der Erwerbsbevölkerung im Aufnahmeland gehören und obwohl einige Aufnahmestaaten auch in die Gesundheit, in die Bildung und in die Sozialleistungen dieser nicht Staatsangehörigen investieren, wurde schon wirtschaftlich festgestellt, dass die Zuwanderer die Sozialleistungen in den Aufnahmeländern nicht überlasten (Brücker u Eger 2012, 177).

Der Schwerpunkt dieser Schrift liegt dabei auf die Berücksichtigung der Sozialleistungen des brasilianischen Staats, d. h., auf die Kosten und/oder Nutzen, die das Migrationsrecht Brasiliens in Bezug auf das Sozialrecht der Migranten ermöglicht.

\section{DER ZUGANG VON MIGRANTEN ZU DEN SOZIALRECHTEN IN BRASILIEN: EIN ÜBERBLICK}

\subsection{DAS PROFIL DER MIGRANTEN IN BRASILIEN HEUTE}

Das größte Land Südamerikas bietet eine vielfältige Bevölkerung: Seit der „Entdeckung” Brasiliens sind Menschen aus allen Kontinenten nach Brasilien ausgewandert. Schon im 16. Jahrhundert lebten zwischen 5 und 10 Millionen Ureinwohner auf dem Gebiet, das heute Brasilien umfasst (Lopes 2009, 275). Ab 1530 (30 Jahre nach der Ankunft von Pedro Álvares Cabral) gab es eine sehr starke Zuwanderung von Portugiesen, die sich während der gesamten portugiesischen Kolonialzeit fortsetzte.

Vom 16. bis zum 19. Jahrhundert war Brasilien zudem stark von der Sklaverei geprägt: im Laufe der Jahrhunderte wurden rund 5 Millionen versklavte Menschen aus Afrika (Lopes 2009, 276) von den Portugiesen dazu gezwungen, nach Brasilien auszuwandern um deren Arbeitskraft in den

\footnotetext{
${ }^{4}$ Der remittance man ist der Emigrant, der aus seinem Heimatland durch Überweisungen finanzielle Unterstützung erhält.
} 
großen Kakao-, Kautschuk-, Zuckerrohr- und Kaffeeplantagen einzusetzen (Pfeifer 1952, 103). Ab dem 19. Jahrhundert kamen Europäer, Menschen aus dem Mittleren Osten und Japaner nach Brasilien und vom Beginn des 20. Jahrhundert an zählten insbesondere Chinesen und andere Südamerikaner zu den größten Gruppen, die nach Brasilien immigrierten (Leão 2013, 101). Die eben kurz beschriebene Vielfältigkeit der Bevölkerung im einzigen portugiesischsprachigen Land Lateinamerikas soll oberflächlich bleiben. Ziel ist es lediglich, einen kurzen Einblick in die ethnische Vielfältigkeit Brasiliens zu gewähren.

Die in Brasilien im Jahre 2010 durchgeführte Volkszählung bestätigt, dass es ab Ende der 2000er Jahre weder Überschuss noch Defizit bei der Bevölkerungsentwicklung Brasiliens gegeben hat. Anders ausgedrückt: Viele brasilianische Staatsangehörige, die in den 80er und 90er Jahren ausgewandert sind, sind zurück nach Brasilien gezogen und viele Staatsangehörige aus den Industrieländern fanden auch in Brasilien ein neues Zuhause - in den 2000er betrug das Saldo der Einwanderer und Auswanderer nahezu Null (de Oliveira 2015, 48) ${ }^{5}$.

Laut den brasilianischen Behörden, die die Bevölkerungsentwicklung verfolgen, wird das Migrationssaldo bis zum Jahr 2035 positiv sein, da Brasilien seit Anfang 2010 immer mehr Zuwanderer angezogen hat (de Oliveira 2015, 49) ${ }^{6}$. Heute lässt sich von einer Zuwanderungswelle sprechen - nicht nur Menschen aus entwickelten Ländern, sondern auch Flüchtlinge suchen in Brasilien eine neue Heimat.

Die unten dargestellte Grafik basiert auf den Daten (Cavalcanti et al. 2015) des brasilianischen Nationalen Rates für Zuwanderung ${ }^{7}$ (Übers. d. Verf.). Sie gibt Auskunft über die Anzahl von Migranten, die zwischen 2011 und 2014 zugelassen worden sind, d. h., die Migranten, die ein vorläufiges und dauerhaftes brasilianisches Wohnvisum bekommen haben:

\footnotetext{
${ }^{5}$ Es ist jedoch nicht zu missachten, dass die Migrationsaussichten von den Vereinten Nationen für den gleichen Zeitraum genau das Gegenteil festgelegt haben; laut den VN ist dieses Saldo negativ, weil die Auswanderung in Brasilien größer als die Zuwanderung sei. Eine Tabelle dieser Berechnung ist verfügbar unter: <https://goo.gl/KavTwA> [Stand: 03.02.2017].

${ }^{6}$ Wichtig ist es zu erwähnen, dass aufgrund der aktuellen politischen und wirtschaftliche Krise, können in den kommenden Jahren sowohl Zuwanderer, als auch Staatsangehörige Brasilien verlassen.

${ }^{7}$ In portugiesischer Sprache: Conselho Nacional de Imigração.
} 


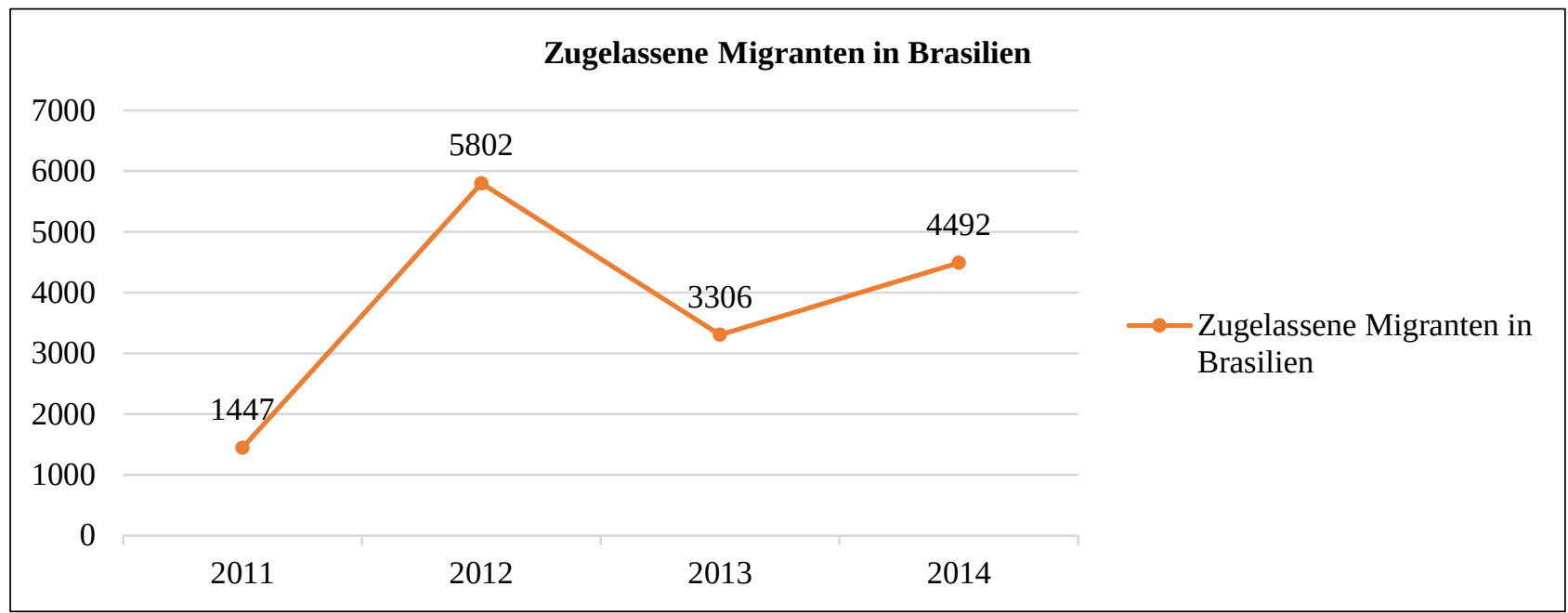

Laut des Büros des Hohen Flüchtlingskommissars der Vereinten Nationen sind 8.863 Menschen bis April 2016 als Flüchtlinge vom brasilianischen Staat anerkannt worden. Das ist die größte Anerkennung des Flüchtlingsstatus in Brasilien überhaupt: Im Jahr 2015 waren 8493 und, im Jahr 2014, $7262^{8}$.

Flüchtlinge und Haitianer scheinen im Übrigen die Mehrheit der derzeitig nach Brasilien kommenden Migranten auszumachen: Etwa 76\% der zwischen 2011 und 2014 in Brasilien zugelassenen Einwanderer sind Migranten aus humanitären Gründen; der restliche Prozentsatz setzt sich aus Menschen zusammen, die aus anderen Gründen nach Brasilien gekommen sind, z.B. zur Gründung einer Familie oder um in Brasilien zu investieren (Cavalcanti et al. 2015, 2):

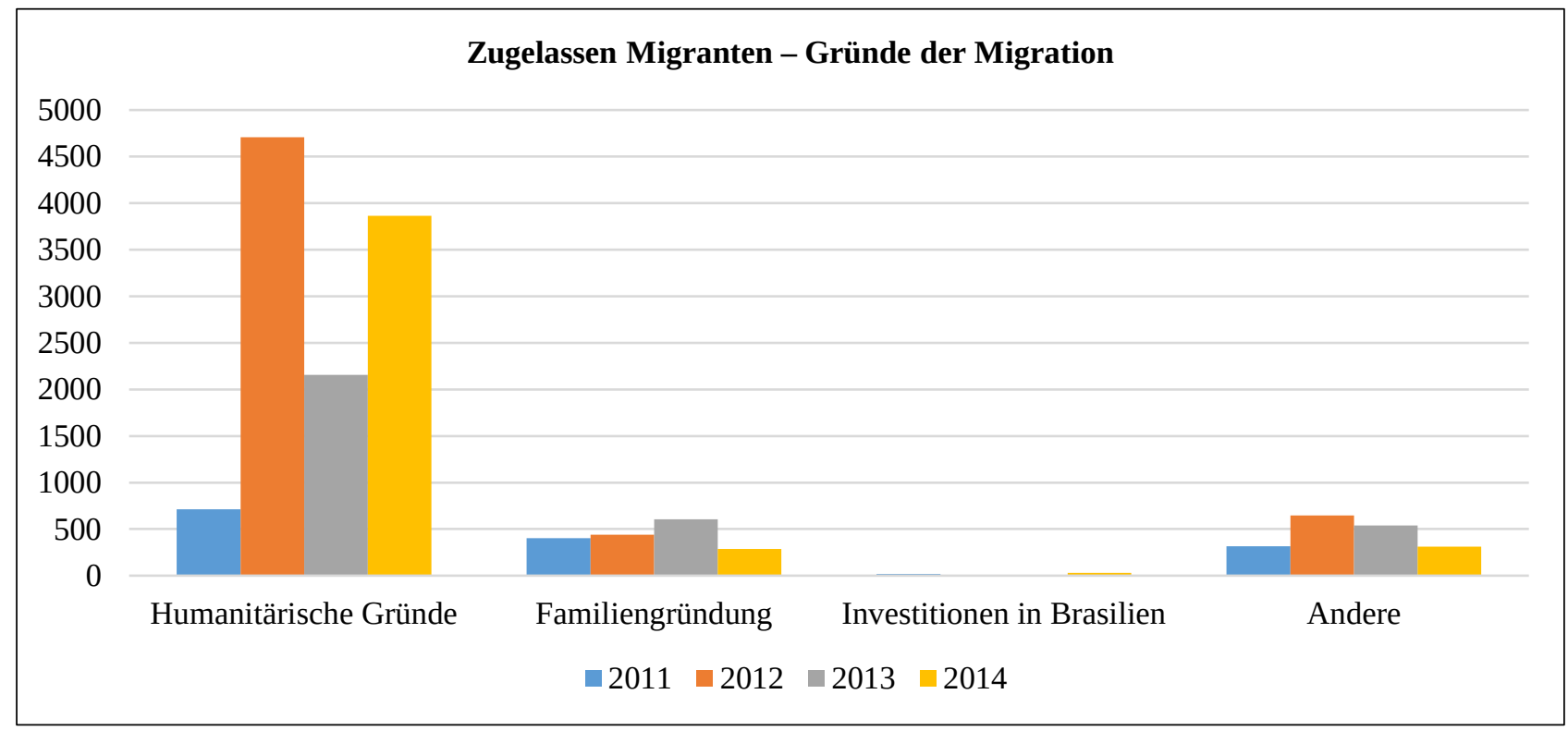

8 Die Daten über Flüchtlinge in Brasilien sind verfügbar unter UNHCRs brasilianische Webseite: $<$ https://goo.gl/UqQTja> [Stand 06.02.2017]. 
Genauso wie viele andere Länder der Europäischen Union beschäftigt sich Brasilien in den letzten Jahren nicht nur mit erwerbstätigen Migranten, sondern auch mit Flüchtlingen, d. h., mit Menschen, die aus politischen, religiösen, wirtschaftlichen, ethnischen Gründen oder aufgrund von Naturkatastrophen ihre Heimat eilig verlassen mussten. Wie und ob Brasilien von dieser Zuwandererwelle profitiert oder ob Brasilien überhaupt dafür bereit ist, wird im Folgenden untersucht.

\subsection{DIE SOZIALRECHTE IN BRASILIEN}

Angesichts der Möglichkeiten von Migranten zu den Rechten in einem bestimmten Gastland vertritt François Rigaux die Meinung, dass das Ausländerrecht unter fünf Perspektiven betrachtet werden sollte: 1) das Recht auf Beitritt, Aufenthalt und Niederlassung; 2) die öffentliche Rechte und die politische Freiheit; 3) das Privatrecht, d. h., die zivilrechtliche Aspekte des Lebens; 4) die wirtschaftliche, soziale und kulturelle Rechte; 5) die politische Rechte und der Zugang zum öffentlichen Dienst (Rigaux 1987, 91).

Das brasilianische Recht folgt der obengenannten französischen Lehre und bestätigt, dass die Rechte der Migranten im Aufnahmeland vom internationalen Privatrecht geregelt werden (Dolinger 2014, 89). Sobald der Aufenthalt und/oder die Niederlassung in einem bestimmten Land ermöglicht wurden, gelten Rechten und Pflichten gegenüber den Migranten. Im brasilianischen Recht werden diese Rechte und Pflichten durch die brasilianische Verfassung, sowie durch das neue brasilianische Migrationsgesetz ${ }^{9}$ geregelt.

In dieser Arbeit werden die Sozialrechte im Fokus stehen. Dies liegt daran, dass einige Migranten hauptsächlich nach einem attraktiven und effektiven Sozialsystem suchen, um sich für ein bestimmtes Land als Aufnahmeland zu entscheiden (Eger u. Weber 2014, 1). Außerdem lassen sich die Migrationskosten, die durch die Gewährung des Zugangs zu Sozialsystemen entstehen, einfacher ausmessen, da sie oft durch öffentliche Statistiken und Zahlen zugänglich sind.

Die brasilianische Verfassung ${ }^{10}$ garantiert in Brasilien eine Gleichbehandlung von Brasilianern und Ausländern. Laut Art. 5 der brasilianischen Verfassung sind alle Menschen vor dem Gesetz gleich, d. h., allen Brasilianern und im Lande wohnhaften Ausländern ${ }^{11}$ wird die

\footnotetext{
${ }^{9}$ Lei no 13.445, vom 24. Mai 2017. Das neue Migrationsgesetz wird im November 2017 in Kraft treten.

${ }^{10}$ Die jetzige Verfassung der Bundesrepublik Brasilien erschien am 5. Oktober 1988 nach einer 21-jährigen Diktaturzeit. Seit ihrer Existenz wurden schon 96 Verfassungsänderungen durchgeführt. Die Verfassung Brasiliens ist verfügbar unter $<$ https://goo.gl/HwJ1Q> [Stand 04.08.2017]

${ }^{11}$ Bei der wortwörtlichen Interpretation des Artikels 5 könnte es darunter verstanden werden, dass nur den in Brasilien wohnhaften Ausländern die Grundrechten garantiert werden. Jedoch erwähnt die Aussage dieses Artikels nur die
} 
Unverletzlichkeit des Rechts auf Leben, Freiheit, Gleichheit, Sicherheit und Eigentum garantiert die sogenannten Grundrechte (Übers. d. Verf.) ${ }^{12}$.

Darüber hinaus hat ein in Brasilien lebender Angehöriger eines anderen Staates schon gemäß des alten brasilianischen Ausländergesetzes, im Art. 95, einen Anspruch auf rechtliche Gleichbehandlung gegenüber brasilianischen Staatsangehörigen (Übers. d. Verf.) $)^{13}$.

Das neue brasilianische Migrationsgesetz aus dem Jahr 2017 bestimmt ebenfalls die Gleichbehandlung von Brasilianern und Ausländern und garantiert in seinem Art. 4 allen Migranten im brasilianischen Territorium ein Recht auf Leben, Freiheit, Gleichberechtigung, Sicherheit und Eigentum.

Die Neufassung des brasilianischen Migrationsgesetzes beendet nunmehr die Diskussion im Hinblick auf den personellen Anwendungsbereich des Gesetzes. Denn es stellt klar, dass alle im brasilianischen Territorium befindlichen Migranten und nicht lediglich die bereits dort wohnhaften Migranten von dem Gesetz erfasst werden.

Aufgrund des Status von Menschenrechten ${ }^{14}$, werden Sozialrechte in vielen Ländern den Migranten garantiert. In Brasilien ist es nicht anders. In diesem Sinne werden allen Ausländern (inklusive Migranten) die Grundrechte garantiert und damit auch die Sozialrechte ${ }^{15}$. Nach der brasilianischen Verfassung sind die Sozialrechte die Rechte auf 1) Bildung; 2) Gesundheit; 3) Nahrung; 4) Arbeit; 5) Wohnung; 6) Freizeit; 7) Sicherheit; 8) Sozialversicherung; 9) Schutz von Mutterschaft und Kindheit; sowie auf 10) Obdachlosenhilfe ${ }^{16}$. Zudem lässt sich sagen, dass die brasilianische Verfassung von garantierten staatlichen Leistungsgewährungspflichten geprägt ist und dass die Verwirklichung dieser Leistungsgewährungspflichten durch aktives staatliches Handeln erfolgen kann.

wohnhaften Ausländer, weil auch die politische Rechte im Artikel 5 aufgezählt werden, d. h., den Zugang zu diesen politischen Rechten haben nur die Ausländer, die in Brasilien wohnhaft sind. Siehe Dolinger, Jacob: Direito Internacional Privado, S.103 ff.

${ }^{12} \mathrm{Im}$ Original in portugiesischer Sprache: Art. $5^{\circ}$ Todos são iguais perante a lei, sem distinção de qualquer natureza, garantindo-se aos brasileiros e aos estrangeiros residentes no País a inviolabilidade do direito à vida, à liberdade, à igualdade, à segurança e à propriedade, nos termos seguintes: [...]

${ }^{13}$ Im Original in portugiesischer Sprache: Art. 95. O estrangeiro residente no Brasil goza de todos os direitos reconhecidos aos brasileiros, nos termos da Constituição e das leis.

${ }^{14}$ Nach dem II. Weltkrieg sind durch die Allgemeine Erklärung der Menschenrechte der Vereinten Nationen die Sozialrechte als Menschenrechte anerkannt worden.

${ }^{15}$ Für eine vertiefte Analyse der Sozialrechte, siehe: Dinamarco, Cândido Rangel: Sobre a tutela jurisdicional do estrangeiro, sowie Bastos, Celso Ribeiro: Comentários à Constituição do Brasil, S. 4.

${ }^{16}$ Im Original in portugiesischer Sprache: Art. $6^{\circ}$ São direitos sociais a educação, a saúde, a alimentação, o trabalho, a moradia, o lazer, a segurança, a previdência social, a proteção à maternidade e à infância, a assistência aos desamparados, na forma desta Constituição. 
Die Rechte auf Bildung, Gesundheit und Sozialversicherung der Ausländer bzw. Migranten in Brasilien werden im Folgenden genauer betrachtet und ebenso einer ökonomischen Analyse unterworfen.

\section{DAS SOZIALRECHT FÜR MIGRANTEN UND DIE NUTZEN UND KOSTEN FÜR DEN BRASILIANISCHEN STAAT}

Im letzten Teil dieser Arbeit werden drei Hauptelemente der Sozialrechte unter einer ökonomischen Perspektive analysiert. Weil eine Analyse aller Sozialrechte den Rahmen dieser Arbeit sprengen würde, wurden drei von ihnen eingehend betrachtet: Das Recht auf Bildung (insbesondere auf ein Hochschulstudium), das Recht auf Sozialhilfe und schließlich das Recht auf Gesundheit unter Einbeziehung verschiedener Formen der Gesundheitsfürsorge.

Im Folgenden werden die Nutzen und Kosten eines Staates bei allen oben erwähnten Rechten berechnet und die gleiche Methode wird danach auf Brasilien angewendet. Um diese Nutzen und Kosten im brasilianischen Recht zu berechnen werden einige Aspekte des brasilianischen Rechts kurz erläutert. Zum Schluss wird geprüft, ob das vorgeschlagene Modell für Brasilien angebracht bzw. erreichbar ist.

Im Hinblick auf die Methode der Berechnung muss erwähnt werden, dass die Kosten des Staates im Vordergrund dieser Arbeit stehen sollen. Erstens, weil die Kosten jedes einzelnen Migranten schlicht und einfach effektiv kaum messbar und zudem meistens sehr subjektiv sind (wie

z. B. die Frage, wie schwer oder einfach es einer Person fällt, eine neue Sprache zu lernen) und zweitens, weil die Gewährleistung von Sozialrechten eine exklusive Leistung des Staates ist.

\subsection{RECHT AUF BILDUNG}

Die Berechnung der Nutzen und Kosten eines Staates gegenüber der Bereitstellung von Bildungsangeboten wird folgendermaßen durchgeführt: die Anzahl der Migranten, die die öffentlichen Universitäten bzw. Hochschulen besuchen und die das Studium in dem Aufnahmeland absolvieren (Kosten), wird mit der Anzahl von Migranten, die die privaten Universitäten bzw. Hochschulen besuchen (Nutzen), verglichen. Aus dem Unterschied zwischen den hieraus errechneten Kosten und Nutzen, wird festgestellt werden können, ob das Recht auf Bildung für ein bestimmtes Aufnahmeland gewinnbringend - somit von Nutzen - ist oder nicht.

Gemäß Art. 206 IV der brasilianischen Verfassung ist in Brasilien der Zugang zu der öffentlichen Bildung gebührenfrei; d. h., dass Schulen und öffentliche Hochschulen/ Universitäten 
vollständig vom brasilianischen Staat finanziert werden ${ }^{17}$. In diesem Sinne wurde durch eine Datenerhebung bei dem Bildungs- und Kulturministerium Brasiliens ${ }^{18}$ festgestellt, wie viele Migranten bzw. Ausländer das Studium in Brasilien von 2009 bis 2013 an öffentlichen und privaten Hochschulen/ Universitäten in Brasilien studiert haben:

\begin{tabular}{c|c|c}
\hline & $\begin{array}{c}\text { Anzahl der Migranten in öffentlichen } \\
\text { Universitäten/Hochschulen }\end{array}$ & $\begin{array}{c}\text { Anzahl der Migranten in privaten } \\
\text { Universitäten/Hochschulen }\end{array}$ \\
\hline $\mathbf{2 0 0 9}$ & 3897 & 10330 \\
\hline $\mathbf{2 0 1 0}$ & 4222 & 8209 \\
\hline $\mathbf{2 0 1 1}$ & 4273 & 7448 \\
\hline $\mathbf{2 0 1 2}$ & 4769 & 7230 \\
\hline $\mathbf{2 0 1 3}$ & 5383 & 7333 \\
\hline
\end{tabular}

Obwohl das Bildungs- und Kulturministerium zwischen Migranten und Ausländern grundsätzlich nicht unterscheidet (in manchen Fällen kann es sich beispielsweise um ein Auslandsjahr/ -semester an einer brasilianischen Universität handeln), wird anhang der Daten deutlich, dass die Mehrheit der Migranten die privaten Universitäten/ Hochschulen in Brasilien besucht. Das bedeutet, dass diesen ausländischen Studierende ihr Studium an diesen Universitäten und die dadurch anfallenden Studiengebühren selbst finanzieren müssen.

2010 wurde von dem brasilianischen Bildungs- und Kulturministerium veröffentlicht ${ }^{19}$, wie viel ein Student den brasilianischen Staat durchschnittlich kostet: in einem Jahr werden pro Student BRL 14.763,00 investiert. Die erwähnte Summe bezieht sich jedoch auf das Jahr 2008.

Zwischen 2009 und 2013 waren 22.544 ausländische Studenten an öffentlichen Hochschulen/ Universitäten in Brasilien eingeschrieben. Bei einer Investition in soeben genannter Höhe pro Student pro Jahr ergibt sich, dass der brasilianische Staat in diesem Zeitraum BRL 332.817.072,00 für das Studium von Ausländern bezahlt hat. Da die Zahlen hinsichtlich ausländischer Studenten an privaten Universitäten/Hochschulen für den gleichen Zeitraum wesentlich höher ausfallen (40.550), kann davon ausgegangen werden, dass Brasilien gesamtwirtschaftlich von den ausländischen Studenten profitiert hat.

\footnotetext{
${ }^{17}$ Im Original in portugiesischer Sprache: Art. 206. O ensino será ministrado com base nos seguintes princípios: [...] IV - gratuidade do ensino público em estabelecimentos oficiais; [...]

${ }^{18}$ Die Bundesregierung in Brasilien ermöglicht es den Bürgern, über ein Webportal nach allen verfügbaren und nicht als Geheim eigestuften Informationen der Bundesregierung zu fragen. Das Zugangssystem für Informationen der Bundesregierung (Sistema de Acesso à Informação) ist unter dem Webportal <https://goo.gl/g9u9I> abrufbar und stellt die Hauptquelle der Datenerhebung dieser Arbeit dar. Die Informationen, die durch das Portal beantragt wurden, wurden direkt dem Verfasser per E-mail zugeschickt und stehen nach Abfrage jedem zur Verfügung.

19 Pressemitteilung des brasilianischen Bildungs- und Kulturministeriums verfügbar unter <https://goo.gl/n7bk1z> [Stand: 06.02.2017].
} 


\subsection{RECHT AUF SOZIALHILFE}

Im Rahmen des Rechts auf Sozialversicherung/Sozialhilfe wird ein ähnliches Modell für die ökonomische Analyse dieses Rechts für Migranten vorgeschlagen. Um die Nutzen und Kosten eines Staates bei der Sozialleistung zu berechnen, müssen zwei Fragen gestellt werden: 1) welcher Anteil des staatlichen Budgets wird für die Sozialleistungen der Migranten/Ausländer ausgegeben?; und 2) wie viel haben Migranten/Ausländer in die Kassen der Sozialversicherungen eines Landes einbezahlt?

Bei dieser Untersuchung werden die Beiträge, die Migranten in die Sozialversicherungen einbezahlt haben, als Nutzen für den aufnehmenden Staat betrachtet. Hinzu kommen indirekt die für den aufnehmenden Staat erwachsenden Vorteile, die durch die Besteuerung der Beitragszahlungen der Migranten an private Sozialversicherungen entstehen.

$\mathrm{Zu}$ den Kosten des Staates zählen dagegen alle möglichen vom Staat angebotenen Sozialleistungen. In diese Kategorie fallen dabei sowohl solche Leistungen, die durch die Beiträge der Migranten selbst gedeckt sind (da dadurch ihre Beiträge wieder aus dem System entfernt werden), und solche, auf die Migranten Anspruch haben, ohne dass sie dafür selbst zahlen müssen.

Es darf nicht übersehen werden, dass die Sozialleistungen sich normalerweise in Form der folgenden Rechte erstrecken: Versicherung von Krankheits-, Invaliditäts- und Todesfällen (durch Arbeitsunfälle, Alter oder Haft verursacht); Schutz der Mutterschaft bzw. der schwangeren Frau; Schutz des unfreiwillig arbeitslosen Arbeitnehmers; Zahlung einer Pension an den Ehegatten des oder der verstorbenen Versicherten; Zahlung einer Pension an den Familienangehörigen eines Strafgefangenen ${ }^{20}$.

Ein weiterer wichtiger Gesichtspunkt dieser Analyse ist, dass viele Länder bilaterale Sozialversicherungsabkommen geschlossen haben. In diesen Abkommen wird für gewöhnlich zwischen den partizipierenden Ländern vereinbart, dass den Staatsangehörigen der beiden Länder jeweils das Recht auf bestimmte Sozialleistungen im jeweiligen anderen Land gewährt wird (Ministério da Previdência Social 2013, 508). Zu diesem Zweck überweist der Staat dem Vertragspartner die Summe, die dem Beitrag für die entsprechenden Sozialleistungen entspricht, sodass der eigene Staatsangehörige im Partnerland bei Bedarf Zugang zu diesen Sozialleistungen erhält (Ministério da Previdência Social 2013, 508)²1. Um dies mit Hilfe des ökonomischen Modells

\footnotetext{
${ }^{20}$ Alle Beispiele haben sich von dem Text der brasilianischen Verfassung inspirieren lassen und können sich je nach Gesetzgebung unterscheiden.

${ }^{21}$ Über die Thematisierung der doppelten Sozialversicherungsabkommen siehe auch Avato, Johana; Koettl, Johannes; Sabates-Wheeler, Rachel: Social Security Regimes, Global Estimates, and Good Practices: The Status of Social Protection
} 
zu untersuchen, müsste ein Vergleich angestellt werden zwischen den Ausgaben eines Landes, welche zum Zwecke der Deckung der Kosten für Sozialleistungen im Partnerland getätigt werden müssen, und den Einnahmen, die der gleiche Staat für die Gewährung analoger Leistungen gegenüber Angehörigen des Partnerstaates erhält.

Brasilien hat beispielsweise eine ganze Reihe von bilateralen Sozialversicherungsabkommen geschlossen, wovon $14^{22}$ bereits in Kraft getreten sind. Daneben wurden ebenfalls multilaterale Sozialversicherungsabkommen geschlossen: eins im Rahmen des Mercosurs (Acordo Multilateral de Seguridade Social do Mercado Comum do Sul - seit 2005) und ein zweites im Kontext der iberoamerikanischen Beziehungen (Convenção Multilateral Iberoamericana de Segurança Social - seit 2011 in Kraft).

Wegen einfacheren Zugangs zur Datenerhebung (Ministério da Previdência Social 2013) kann eine zahlenmäßige Analyse im Rahmen der von Brasilien geschlossenen Bilateralen/ Multilateralen Sozialversicherungsabkommen durchgeführt werden. Laut des brasilianischen Ministeriums für Sozialhilfe hat Brasilien zwischen 2011 und 2013 den Vertragspartnern der Bilateralen bzw. Multilateralen Sozialversicherungsabkommen insgesamt BRL 3.014.000,00 (Ministério da Previdência Social 2013, 510-511) überwiesen, um die Kosten der Sozialleistungen der brasilianischen Bürger im Ausland zu decken. Selbstverständlich sind auch Zahlungen durch die Vertragsstaaten an Brasilien erfolgt, damit diese in Brasilien in den Genuss eben jener Sozialleistungen kommen.

Jedoch sind die dem Verfasser in diesem Fall zur Verfügung stehenden Daten unzureichend, um darauf schließen zu können, ob die Zahlungen von den Staaten ausgeglichen und in jeweils ähnlicher Höhe getätigt worden sind oder ob es zu Verschiebungen zugunsten oder zulasten Brasiliens oder eines seiner Vertragspartner gekommen ist.

\subsection{RECHT AUF GESUNDHEIT}

Das vorgeschlagene Modell für die Analyse des Migrationsrechts in Bezug auf das Recht auf Gesundheit besteht aus der Berechnung der Nutzen und Kosten, die durch die Gesundheitsversorgung von Migranten entstehen. Dafür sollen die Ausgaben des Staates für die

for International Migrants, in: World Development 38 (2010) 4, S. 455-466 und Bolderson, Helen; Roberts, Simon: Social security across frontiers, in: New Global Development 13 (1997) 1, S.7-23.

${ }^{22}$ Mit den folgenden Ländern bzw. Regionen: Deutschland, Belgien, Kapverdische Inseln, Kanada, Chile, Spanien, Frankreich, Griechenland, Italien, Japan, Luxemburg, Portugal, Südkorea, Québec. Diese Information ist verfügbar unter: $<$ https://goo.gl/MDYjnx> [Stand: 03.02.2017]. 
Gesundheitsversorgung der Migranten mit den Einnahmen, die durch Beiträge der Migranten zu privaten Krankenkassen erzielt werden, verglichen werden. Anders ausgedrückt: Wie viele Migranten nehmen einerseits die staatliche Gesundheitsversorgung in Anspruch und verlassen sich dadurch auf öffentliche Leistungen und wie viele entscheiden sich andererseits zum Eintritt in private Krankenversicherungen, wodurch sie die Beiträge selbst übernehmen müssen.

Die Analyse dieses Punktes ist in Brasilien deswegen besonders und unterscheidet sich von anderen Ländern, da es das sogenannte ‘einheitliche Gesundheitssystem’ (Sistema Único de Saúde SUS) gibt. Das System wird von der Regierung finanziert (zum größten Teil von der Bundesregierung, aber auch von den Bundesstaaten und Städten ${ }^{23}$ ) und erlaubt allen Brasilianern und Ausländern den kostenlosen Zugang zu aller Art medizinischer Behandlungen²4.

Aufgrund struktureller Probleme im öffentlich finanzierten Gesundheitssektor, der sich beispielsweise durch einen hohen Mangel an Ärzten und unzureichend zur Verfügung stehenden medizinischen Geräten ausdrückt, verlassen sich viele Brasilianer und Migranten aber lieber auf eine privat finanzierte Gesundheitsversorgung.

In diesem Zusammenhang wurden zwei Daten bei dem Gesundheitsministerium über das Zugangssystem für Informationen der Bundesregierung beantragt, um die Nutzen und Kosten des brasilianischen Staates zu berechnen: 1) wie viel hat es in den letzten 4-5 Jahren dem brasilianischen Staat gekostet, dass Migranten die staatliche Gesundheitsversorgung nutzen und 2) wie viel haben die Migranten in den letzten 4-5 Jahren in Brasilien in private Krankenkassen einbezahlt, um sich die Gesundheitsversorgung im Land zu garantieren.

Die Antwort des Gesundheitsministeriums war für die vorliegende Untersuchung leider wenig zielführend, da sich das Ministerium darauf beruft, dass das brasilianische „einheitliche Gesundheitssystem” die Kosten für die medizinische Behandlung von Migranten, Ausländern und/ oder Flüchtlingen nicht erhebt. Erstens, weil bei der Behandlung von kranken Menschen in den öffentlichen Krankenhäusern und/ oder Kliniken die Herkunft der Migranten oder der im Lande wohnhaften Ausländer nicht nachgefragt werden und dementsprechend nicht registriert werden. Zweitens, weil das Recht auf Gesundheit in Brasilien vollständig ist, d. h., dass allen Brasilianern und Ausländern der kostenlose Zugang zu aller Art medizinischer Behandlungen erlaubt ist und aus

\footnotetext{
${ }^{23}$ Gemäß Artikel $198 \S 1^{\circ}$ Brasilianische Verfassung.

${ }^{24}$ Gemäß Artikel 196 Brasilianische Verfassung in Verbindung mit Art. 6 Brasilianische Verfassung.
} 
diesem Grund wird es im Moment der medizinischen Behandlung nicht unterscheidet, wer eine brasilianische oder ausländische Staatsangehörigkeit hat ${ }^{25}$.

Auch hinsichtlich der Frage nach der Anzahl der Migranten, die in den letzten 4-5 Jahren in die privaten Krankenkassen einbezahlt haben, ist kein anderes Ergebnis zu erzielen. Die Nationale Agentur für Gesundheit (Agência Nacional de Saúde - ANS) registriert die Staatsangehörigkeit der durch private Krankenkassen Versicherten nicht ${ }^{26}$.

Als Folge dieses Informationsmangels ist es nicht möglich, das Modell für die ökonomische Analyse des Rechts auf Gesundheit auf Brasilien anzuwenden. Es kann daher nicht beurteilt werden, ob es für den brasilianischen Staat im Rahmen der medizinischen Versorgung von Migranten eher zu Kosten oder Nutzen kommt.

\section{SCHLUSSBETRACHTUNG}

Das Ziel dieser Arbeit war es, ein passendes Modell für eine ökonomische Analyse des Migrationsrechts (im Rahmen der sozialen Rechte von Migranten) vorzuschlagen und zu ermitteln, ob und in wie fern dieses Modell auf Brasilien anwendbar ist.

Dazu erfolgte zuerst eine allgemeine Beschreibung der Grundbegriffe, die das Thema umfassen. Hier wurde festgelegt, dass die Migrationen als einer der wichtigsten Strukturfaktoren der globalen Wirtschaft anerkannt werden und dass die Begriffe „Migranten” und „Ausländer” oft verwechselt werden. In diesem Zusammenhang wurde die Situation der Migranten in Brasilien beschrieben: hier wird deutlich, wie groß die Anzahl von Migranten in dem Land ist und wie bedeutungsvoll die Aufnahme von Flüchtlingen und Arbeitssuchenden wird, obwohl immer noch viele Brasilianer ins Ausland gehen. Weiterhin wurde dargelegt, dass die Migrationskosten vielfältig sind, weil sie aus verschiedenen sozialen, kulturellen und ökonomischen Faktoren bestehen.

\footnotetext{
${ }^{25}$ Die originelle Version der Antwort in portugiesischer Sprache (verfügbar auch im persönlichen Archiv des Verfassers): „[...] Após algumas consultas internas confirmei a informação de que não há como levantar o gasto exclusivo com atendimento de imigrantes ou refugiados no SUS. O primeiro argumento é de ordem conceitual. O caráter universal do sistema não restringe o atendimento, mas o registro referente à nacionalidade ou situação legal de residente estrangeiro não é realizado. A falta de registro nos sistemas de informação do SUS é um problema que impede a consolidação de dados.

O sistema atende, porém não realiza ajustes para captar essas informações no momento do atendimento.

O direito à saúde no Brasil é considerado pleno e não está restrito ao status migratório, e por conta disso, não há como vincular gasto com refugiados e migrantes. [...]”

${ }^{26}$ Die originelle Version der Antwort in portugiesischer Sprache (verfügbar auch im persönlichen Archiv des Verfassers): „[...] Conforme informadao [sic] pela Diretoria de desenvolvimento Setorial (DIDES), Esta Agência não possui a informação solicitada. A ANS não tem em seus sistemas a nacionalidade dos beneficiários de forma a possibilitar a extração dessa informação solicitada. [...]”
} 
Nach einem Überblick der Sozialrechte für Migranten in Brasilien wurden im letzten Kapitel drei Hauptelemente der Sozialrechte unter einer ökonomischen Perspektive analysiert. Drei Sozialrechte wurden unter die Lupe genommen: Das Recht auf Bildung - insbesondere die Hochschulstudien -; das Recht auf Sozialhilfe; und schließlich das Recht auf die Gesundheit.

Im Anschluss daran wurden der Nutzen und die Kosten des brasilianischen Staates mit Hilfe des vorgeschlagenen Modells berechnet. Durch Daten, die von der brasilianischen Regierung zur Verfügung gestellt worden sind, wurde die Berechnung der Migrationskosten durchgeführt. Anhand der vorhandenen Forschungsergebnisse konnte gezeigt werden, dass sich der brasilianische Staat nicht sicher sein kann, ob sich die Einwanderungswelle positiv oder negativ auf seine Wirtschaft auswirkt. Der Grund dafür ist, dass die Regierung die für die Bereitstellung solcher Rechte anfallenden Kosten nicht registriert, d. h. die brasilianische Regierung weiß nicht, wie viel Geld sie für die Gesundheitsvorsorge, Bildung und Sozialleistungen an Migranten ausgibt. Dies liegt daran, dass Brasilien es nicht gesondert vermerkt und dokumentiert, wenn ein Migrant/ Ausländer öffentliche Versorgungsleistungen in Anspruch nimmt.

Um eine effektive Migrationspolitik betreiben zu können, müsste sich der brasilianische Staat aber im Klaren darüber sind, was genau die Kosten der Ein- und Auswanderungsbewegungen sind, die Brasilien betreffen. Er müsste wissen, wie viele Migranten durchschnittlich die Angebote der brasilianischen Versorgungsbetriebe in Anspruch nehmen und wie viel der Staat dadurch in Migranten investiert. Ohne sich über diese Kosten und die ökonomische Seite der Zuwanderung im Klaren zu sein, kann der brasilianische Staat die Migration nicht effektiv durch Gesetze lenken, da er sich über die Folgen von Gesetzesänderungen (insbesondere über ihre Kosten und Nutzen) nicht im Klaren ist. Die Migrationskosten und die ökonomische Seite der Zuwandererwelle spielen eine große Rolle bei der Organisation eines Staates und bei dem Entwurf neuer Gesetze.

Im Hinblick auf eine weitere Ausarbeitung dieser Thematik wären genauere Daten nötig. Darüber hinaus wäre es interessant zu untersuchen, ob diese vorgeschlagenen Modelle auch in Deutschland anwendbar wären und zu einem konkreten Ergebnis führen würden. 


\section{LITERATURVERZEICHNIS}

Avato, Johana, Johannes Koettl und Rachel Sabates-Wheeler. 2009. "Social Security Regimes, Global Estimates, and Good Practices: The Status of Social Protection for International Migrants”. World Development 38 (4): 455-466.

Bastos, Celso Ribeiro. 1988. Comentários à Constituição do Brasil: promulgada em 5/10/1988. São Paulo: Saraiva.

Bayer, Christian und Falko Juessen. 2012. "On the dynamics of interstate migration: Migration costs and self-selection”. Review of Economic Dynamics 15: 377-401.

Bolderson, Helen und Simon Roberts. 1997. "Social security across frontiers". New Global Development 13 (1): 7-23.

Brücker, Herbert und Thomas Eger. 2012. "The law and economics of the free movement of persons in the European Union” In Research Handbook on the Economics of European Union Law, 146179. Cheltenham: Edward Elgar.

Cavalcanti, Leonardo, Tânia Tonhati und Antônio Tadeu Oliveira, Hrsg. 2015. Autorizações concedidas a estrangeiros. Brasília: Relatório 2015.

Clarke, Charles. 2011. The EU and migration. A call for action. London: Centre for European Reform.

Collier, Paul. 2015. Exodus - Warum wir Einwanderung neu regeln müssen. Bonn: Bundeszentrale für politische Bildung.

Cox, Adam B. und Eric A Posner. "The second-order Structure of Immigration Law”. Stanford Law Review 59 (4): 809-856.

Dinamarco, Cândido Rangel. 2002. "Sobre a tutela jurisdicional do estrangeiro”. Revista de Processo: RePro. 27 (107): 248-251.

Dolinger, Jacob. 2014. Direito Internacional Privado. Parte Geral. Rio de Janeiro: Editora Forense.

Eger, Thomas und Franziska Weber. 2014. "Immigration law" In Encyclopedia of Law and Economics, hrsg. V. Jürgen Georg, 1-11. New York: Backhaus.

Încalţăău, Cristian, Sorin-Ştefan Maha und Liviu-George Maha. 2011. “A broader look on migration - A two way interaction between development and migration in the country of origin". Review of Economic \& Business Studies 4 (2): 285-297.

International Organization for Migration. 2012. Economic Cycles, Demographic Change and Migration. Genf: International Organization for Migration.

Kälin, Walter und Jörg Künzli. 2008. Universeller Menschenrechtsschutz. Basel: Helbing Lichtenhahn. 
Leão, Augusto Veloso. 2013. "Public Policy and Media Frames. The debate over migration in Brazil”. Brazilian Political Science Review 7 (1): 94-117.

Lopes, Cristiane Maria Sbalqueiro. 2009. Direito de Imigração. O Estatuto do Estrangeiro em uma perspectiva de direitos humanos. Porto Alegre: Nuria Fabris Editora.

Michaelides, Marios. 2011. "The effect of local ties, wages, and housing costs on migration decisions”. The Journal of Socio-Economics 40: 132-140.

Ministério da Previdência Social, Empresa de Tecnologia e Informações da Previdência Social 2013. Anuário Estatístico da Previdência Social, vol. 22. Brasília: MPS/DATAPREV.

Oliveira, Antônio Tadeu de. 2015. "O perfil geral dos imigrantes no Brasil a partir dos censos demográficos 2000 e 2010”. Cadernos Obmigra - Revista Migrações Internacionais 1 (2): 48-73.

Pfeifer, Gottfried. 1952. „Brasiliens Stellung in der kulturgeographischen Entwicklung der neuen Welt (Teil I)”. Erdkunde 6 (2/3): 85-103.

Rigaux, François. 1987. Droit International Privé. Tome I. Théorie générale. Brüssel: Maison F. Larcier.

Ronzani, S. 1979. Migration als individuelle und als gesellschaftliche Problemlösungsstrategie, unv. Diss., Handelshochschule St. Gallen.

Straubhaar, Thomas. 1988. On the Economics of International Labor Migration. Bern und Stuttgart: Verlag Paul Haupt.

Sykes, Alan O. 1992. "The Welfare Economics of Immigration Law. A Theoretical Survey with an Analysis of U.S. Policy”. Coase-Sandor Institute for Law \& Economics, Working Paper No. 10: 1-46.

Thomas, Brinley. "Migration (Economic Aspects)”. In International Encyclopedia of the Social Sciences, hrsg. V. Sills, D.L., 292-300. 10. Aufl. New York [et al]: Crowell Collier and Macmillan.

Tiburcio, Carmen. 2001. The human Rights of Aliens under International and Comparative Law. The Hague: Martinus Nijhoff Publishers.

United Nations - Department of Economic and Social Affairs. 2014. International Migration 2013. New York: United Nations.

United Nations Statistics Division. 1998. Recommendations on Statistics of International Migration, Revision 1. New York: United Nations.

Zelinsky, W. 1971. “The Hypothesis of the Mobility Transition”. Geographical Review 61: 219249. 
Emília Lana de Freitas Castro Doutoranda em Direito Internacional na Universität Hamburg (2015-2018). Bolsista de doutorado da Coordenação de Aperfeiçoamento de Pessoal de Nível Superior (Capes), fundação do Ministério da Educação (MEC). Mestre em Direito Internacional pela Universidade do Estado do Rio de Janeiro (2014). Bacharel em Direito pela Universidade do Estado do Rio de Janeiro (2011). Membro da Associação de Juristas Alemanha-Brasil (DBJV), membro pleno da Asociación Americana de Derecho Internacional Privado (ASADIP) e membro de Netzwerk Migrationsrecht (Alemanha). Advogada. E-mail: Emilia.Castro@studium.uni-hamburg.de

Raphael Carvalho de Vasconcelos

Professor Adjunto de Direito Internacional Público na Universidade do Estado do Rio de Janeiro. Professor Adjunto de Direito Público na Universidade Federal Rural do Rio de Janeiro.E-mail: rvasconcelos@raphaelvasconcelos.com 